\title{
Kardinalen potentziez eta Shelahren PCF aieruaz
}

Juan Carlos Martínez

Matematika eta Informatika Fakultatea (Universitat de Barcelona)

\section{(On powers of cardinals and Shelah's PCF conjecture)}

Larraitz Zubeldiak ingelesetik itzulia.

DOI: $10.1387 /$ gogoa.20365

\begin{abstract}
We describe some open problems related to PCF theory.

Keywords: powers of cardinals, PCF theory, compact scattered spaces.
\end{abstract}

\section{Laburpena}

PCF teoriarekin erlazionatuta argitzeke dauden arazo batzuk deskribatzen ditugu.

Gako-hitzak: kardinalen potentziak, PCF teoria, espazio trinko sakabanatuak.

Continuumaren Hipotesia da $2^{\omega}=\omega_{1}$ delako enuntziatua, zeinak esan nahi duen zenbaki naturalen azpimultzo guztien bildumaren kardinalitatea lehenbiziko kardinal zenbatezina dela. Continuumaren Hipotesia lehenbiziko problema zen Hilbertek, 1900ean, argitaratutako 23 problemako zerrenda ezagunean. Eta Orokortutako Continuumaren Hipotesia da $\kappa$ kardinal infinitu ororentzat $2^{\kappa}=\kappa^{+}$delako enuntziatua (zeinak esan nahi duen $\kappa$-ren azpimultzo guztien bildumaren kardinalitatea $\kappa$-ren ondorengo kardinala dela). Gödelek frogatu zuen, Unibertso Eraikigarrian, betetzen dela Orokortutako Con- 
tinuumaren Hipotesia. Ezaguna da, baita ere, $\kappa$ kardinal infinitu ororentzat, $2^{\kappa}=\kappa^{+}$den edo ez ZFCtik independentea dela. $\kappa$ kardinal infinitu batentzat $2^{\kappa}=\kappa^{+}$ote den galderari buruzko informazio partziala Königen Teoremak ematen $\mathrm{du}$, zeinak baiezten duen, $\kappa$ kardinal infinitu ororentzat, $2^{\kappa}$-ren kofinalitatea $\kappa^{+}$dela gutxienez. Hain zuzen, Königen Teorema da kardinal erregularrei buruz esan dezakegun guztia; izan ere, Eastoni zor zaion oinarrizko teoremaren arabera, V-k Orokortutako Continuumaren Hipotesia asetzen badu, orduan $\mathrm{f}:$ ORD $\rightarrow$ ORD funtzio monotono ororentzat, non $\alpha<\mathrm{f}(\alpha)$ eta $x_{\alpha}<\operatorname{cof}\left(x_{\mathrm{f}(\alpha)}\right), \alpha$ ordinal bakoitzarentzat V-ren kardinalari eusten dion estentsio generiko bat dagoen, non $2^{\varkappa \alpha}=x_{\mathrm{f}(\alpha)}$ den $\alpha$ ordinal ororentzat, non $x_{\alpha}$ erregularra den. Beraz, ageriko baldintza batzuk asetzen dituen ezein jokaera kardinal aritmetiko gauza daiteke kardinal erregularretako potentziafuntzioaren jokaera gisa. Hala ere, oinarrizko emaitza hori ezin heda daiteke kardinal singularren klasera, zeren 1980ko hamarkadaren amaieran Shelahk emaitzen sail bat frogatu zuen, eta kardinal borneak lortu zituen potentziafuntzioak kardinal singularretan duen jokaeran, delako kardinal singularraren azpiko kardinal erregularren emaitza mugatuak aztertuta. Horrek PCF esaten zaion teoriara (kofinalitate posibleen teoriara) eraman zuen, zeinak ondorio garrantzitsu eta espero gabeko asko dituen kardinalen aritmetikan eta zeinak, halaber, aplikazio interesgarriak izan zituen aljebran eta topologian (ikus [1], [2], [4], [13] eta [14]).

Gogoratu $\lambda$ kardinala muga gogorreko kardinala dela, baldin eta $2^{x}<\lambda$ bada $x<\lambda$ kardinal ororentzat. Eta gogoratu $x_{\alpha}$ kardinal infinitua ( $x$ funtzioaren) puntu finkoa dela, baldin eta $x_{\alpha}=\alpha$. Orobat, $\alpha$ ordinal bat bada eta $\mathrm{n} \in \omega, \mathrm{n}>1$ izanik, $\alpha^{+}$-ren bidez denotatzen dugu $\alpha$-ren ondorengo kardinala eta $\alpha^{+n}$-ren bidez denotatzen dugu $\alpha^{+\ldots n \ldots+}$ kardinala. Hortaz, honako emaitza esanguratsu hau frogatu zuen Shelahk [14]n:

1. teorema. Demagun $\lambda=x_{\alpha}$ puntu finkoa ez den kardinal singular bat dela. $\lambda$ muga gogorreko kardinal bat bada, orduan $2^{\lambda}<x_{\alpha+4}$.

Kasu interesgarriena da 1 . teoremako $\lambda$ kardinala $x_{\omega}$ kardinal gutxienik singularrena denean. Honako emaitza hau da, orduan, goiko teoremaren berehalako ondorioa.

2. teorema. $x_{\omega}$ muga gogorreko kardinala bada, orduan $2^{\varkappa \omega}<x_{\omega 4}$.

Shelahek, Gitikek, Magidorrek, Woodinek eta beste batzuek emaitza esanguratsuak lortu dituzten arren, inork ez daki goiko borneak hobetu daitezkeen.

PCF teorian, objektu nagusietako bat PCF eragilea da. Hasteko, ohartu A kardinalen multzo infinitua bada, D ultrairagazki bat bada A-n eta ПA-k A domeinua duen $f$ funtzio guztien multzoa denotatzen badu, non $f(a) \in a$ den a $\epsilon$ A guztientzat, orduan ПA linealki ordenatuta dago honako erlazio honen bidez: $\mathrm{f} \leq_{\mathrm{D}} \mathrm{g}$ bsb $\{\delta \in \mathrm{A}: \mathrm{f}(\delta) \leq \mathrm{g}(\delta)\} \in \mathrm{D}$. Beraz, linealki ordenatutako 
multzo horrek ondo zehaztutako kofinalitatea du, zeina cof(ПA/D)-ren bidez denotatuko den. Hala, A kardinal erregularren multzo infinitua bada, definitzen dugu

$$
\operatorname{PCF}(A)=\{\operatorname{cof}(\Pi A / D): D \text { ultrairagazki bat da A-n }\}
$$

A multzo bat kardinal erregularren tarte bat dela esaten dugu, baldin eta A kardinalen tarte baten eta kardinal erregularren klasearen arteko ebakipuntua bada. Eta A progresiboa dela esaten dugu, baldin eta A infinitua bada eta $|\mathrm{A}|<\min (\mathrm{A})$. Orduan, 1. teorema frogatzeko, Shelahk erakutsi zuen A kardinal erregularren tarte progresibo bat bada, orduan $\mathrm{PCF}(\mathrm{A})$ elementu maximoa duen kardinal erregularren tarte bat dela eta $|\mathrm{PCF}(\mathrm{A})|<|\mathrm{A}|^{+4}$. Honako galdera hau da aritmetika kardinalean argitzeke dagoen arazo nagusietako bat.

1. galdera. Demagun A kardinal erregularren tarte progresiboa dela. Egia al da $|\mathrm{PCF}(\mathrm{A})|=|\mathrm{A}|$ ?

Kardinal erregularren ezein A tarte progresiborentzat $|\mathrm{PCF}(\mathrm{A})|=|\mathrm{A}|$ delako enuntziatuari PCF Aierua esaten zaio. Aierua egiazkoa balitz, hobetu egingo genuke 1 . teoremaren $2^{\lambda}$-ko bornea $x_{\alpha+}$-ra, eta, hala, hobetu egingo genuke 2 . teoremaren $2^{\varkappa \omega}$-ko bornea $x_{\omega 1}$-era. Borne hori hobeezina litzateke, Magidorri eta Shelahri zor zaizkien funtsezko emaitzei esker bai baitakigu, kardinal handiak badirela emantzat joz, multzo-teoriaren ereduak sor ditzakeela batek, non $x_{\omega}$ muga gogorreko kardinala den eta $2^{\varkappa \omega}=x_{\alpha+1}$ den $\alpha$ ordinal arbitrario infinitu zenbakarri batentzat (ikus [5]).

Halaber, [6] eta [11]ra igortzen dugu irakurlea PCF eragileak kardinal handiei dagokienez dituen emaitza eta aplikazio batzuk ikusteko.

Bestetik, gauza jakina da A kardinal erregularren tarte progresibo bat bada, PCF eragilea PCF(A)-ren azpimultzoetako itxitura-eragiketa bat dela, halako eran, non horri lotutako PCF(A) azpiko multzoaren espazio topologikoak ezaugarri garrantzitsu batzuk asetzen dituen, zeinak Shelahk erabili zituen $|\mathrm{PCF}(\mathrm{A})|<|\mathrm{A}|^{+4}$ dela frogatzeko. Gogoan izan X espazio topologiko bat sakabanatua dela, baldin eta X-ren azpiespazio ez-huts orok puntu isolatu bat badu. Orduan jakina da kardinal erregularren A tarte progresibo ororentzat PCF(A)-rekin erlazionatutako espazio topologikoa trinkoa, Hausdorff eta sakabanatua dela (ikus [2] eta [14]).

LCS espazioaren bidez adierazi nahi dugu lokalki trinkoa, Hausdorff eta sakabanatua den espazioa. X LCS espazio bat bada eta $\alpha$ ordinal bat bada, X-ren ath Cantor-Bendixson maila honako honen bidez definitzen da: $\mathrm{I}_{\alpha}(\mathrm{X})=\mathrm{X} \backslash \mathrm{U}\left\{\mathrm{I}_{\beta}(\mathrm{X}): \beta<\alpha\right\}$-ren puntu isolatuen multzoa. X-ren altuera definitzen dugu $h t(X)=\alpha$ gutxienik ordinalena gisa, non $I_{\alpha}(X)$ finitua den. Argi dago X LCS espazio ez-trinko baten puntu bateko trinkoketa Y espazio 
trinko, Hausdorff eta sakabanatu bat dela, non $\mathrm{ht}(\mathrm{Y})=\mathrm{ht}(\mathrm{X})$ eta $\mathrm{I}_{\alpha}(\mathrm{Y})=\mathrm{I}_{\alpha}(\mathrm{X})$ diren $\alpha<\mathrm{ht}(\mathrm{X})$ ororentzat. X LCS espazio bat bada, $<\left|\mathrm{I}_{\alpha}(\mathrm{X})\right|: \alpha<\mathrm{ht}(\mathrm{X})>$ segidari X-ren segida kardinala esaten zaio. [8] laburpen-artikuluan aurki dezake irakurleak LCS espazioentzako segida kardinalen emaitzen zerrenda zabala.

Honako nozio hau, zeinak ahalbidetzen digun LCS espazioak zuzenean ordena partzialetatik eraikitzea, tresna erabilgarria da PCF eragilea aztertzeko.

1. definizioa. (a) Demagun $\mathrm{T}=\mathrm{U}\left\{\mathrm{T}_{\alpha}: \alpha<\eta\right\}$ dela $\eta$ ordinal ez-zeroren batentzat, non $\mathrm{T}_{\alpha}$ bakoitza multzo ez-huts bat den eta $\mathrm{T}_{\alpha} \cap \mathrm{T}_{\beta}=\varnothing$ den $\alpha<\beta<\eta$-rentzat. Izan bedi $\leq^{*}$ ordena partzial bat T-n. $t \in \mathrm{T}$ ororentzat, $\mathrm{b}_{\mathrm{t}}$-ren bidez denotatzen dugu $\left\{\mathrm{s} \in \mathrm{T}: \mathrm{s} \leq^{*} \mathrm{t}\right\}$ multzoa. Orduan esaten dugu $\left(\mathrm{T}, \mathrm{s}^{*}\right) L C S$ pomultzo bat dela, baldintza hauek asetzen badira:

(1) Baldin eta $s<<^{*} t, s \in T_{\alpha}$ eta $t \in T_{\beta}$, orduan $\alpha<\beta$.

(2) Baldin eta $t \in T_{\beta}$ eta $\alpha<\beta$, orduan $\left\{s \in T_{\alpha}: s<^{*} t\right\}$ infinitua da.

(3) $s$ eta $t$ T-ren elementu desberdinak baldin badira, $u_{1}, \ldots, u_{n} \in T$ elementu asko daude finituki, non $b_{s} \cap b_{t}=b_{u 1} U \ldots U b_{u n}$.

(b) Baldin eta $\left(\mathrm{T}, \leq^{*}\right) \mathrm{T}=\mathrm{U}\left\{\mathrm{T}_{\alpha}\right.$ : $\left.\alpha<\eta\right\}$-dun LCS pomultzo bat bada, honela definitzen dugu $\left(\mathrm{T}, \leq^{*}\right)$-rekin erlazionatutako espazioa. Bere azpiko multzoa $T$ da. Eta $s \in T$ ororentzat, s-ren oinarrizko ingurune bat $\mathrm{b}_{\mathrm{s}} \backslash\left(\mathrm{b}_{\mathrm{u} 1} \mathrm{U} \ldots \mathrm{U} \mathrm{b}_{\mathrm{un}}\right)$ formako multzo bat $\mathrm{da}$, non $\mathrm{n}<\omega$ eta $\mathrm{u}_{1}, \ldots, \mathrm{u}_{\mathrm{n}}<^{*} \mathrm{~s}$. Erraza da egiaztatzea sortzen den $\mathrm{X}$ espazio topologikoa espazio lokalki trinkoa, Hausdorf eta sakabanatua dela, non $\mathrm{I}_{\alpha}(\mathrm{X})=\mathrm{T}_{\alpha}$ den $\alpha<\eta$ ororentzat. Y T-ren azpimultzo bat bada, $\bar{Y}$-ren bidez denotatzen dugu Y-ren itxitura X espazioan.

Orain, LCS pomultzo baten nozioaren fintzea aurkeztuko dugu, Magidor eta Foremani zor zaiena, zeinetan baldintza batzuk eransten diren PCF eragilearen oinarrizko propietateak islatzeko.

2. definizioa. Izan bedi $\kappa$ kardinal erregular bat. $\kappa-P C F$ egitura bat $\left(\theta+1, \leq^{*}\right)$ LCS pomultzo bat da, non $\theta$ ordinal infinitu bat den, non honako baldintza hauek asetzen diren:

(PCF1) Baldin eta $\mu<^{*} \xi$, orduan $\mu \epsilon \xi$.

(PCF2) $\bar{\kappa}=\theta+1$.

(PCF3) Baldin eta I $\theta+1$-en dagoen tarte bat bada, orduan I ere tarte bat da.

(PCF4) $\xi \in \theta$ ororentzat, $\xi<^{*} \theta$.

(PCF5) Kofinalitate zenbatezinaren $\mu \in \theta$ bakoitzarentzat, bada $\mu$-ren $C_{\mu}$ azpimultzo itxi bornegabe bat non $\mathrm{C}_{\mu} \mu+1$-en dagoen.

[14]ko honako emaitza esanguratsu honetan, Shelahk frogatu zuen PCF axiomak egiazkoak direla. 
3. teorema. Izan bedi $\kappa$ kardinal erregular bat. Izan bedi A $\kappa$ ordena motako kardinal erregularren tarte progresibo bat. Orduan ordena partzial bat dago PCF(A)-n, zeinak PCF(A) к-PCF egitura bat bihurtzen duen.

P к-PCF egitura oro LCS pomultzo bat denez, bada X LCS espazio bat P-ri lotua 1 (b) definizioaren zentzuan. Eta (PCF4) baldintzaren arabera, X trinkoa da. Orduan A $\kappa$ ordena motako kardinal erregularren tarte progresibo bat bada $\kappa$ kardinal erregularrarentzat, eta $\leq^{*} \mathrm{PCF}(\mathrm{A})$-ko ordena partzial bat bada, 3. teoremak emandakoa, $\mathrm{X}\left(\mathrm{PCF}(\mathrm{A})\right.$ )-ren bidez denotatzen dugu (PCF(A), $\left.\leq^{*}\right)$-ri lotutako espazio trinko, Hausdorff eta sakabanatua.

$\kappa$ kardinal erregular bat bada, esaten dugu X $\kappa$-PCF espazio bat dela, baldin eta $\mathrm{P}$ к-PCF egitura bat bada non X P-ri lotutako espazio tipologiko bat den. Eta X PCF espazio bat da, baldin eta X $\kappa$-PCF espazio bat bada $\kappa$ kardinal erregular batzuentzat. Hurrengo arazo orokorra PCF teorian argitzeke dagoen galdera nagusietako bat da.

2. galdera. Zein PCF espazio existitzen dira konsistenteki?

2. galderari dagokionez, Shelahk frogatu zuen $\kappa$ kardinal erregular bat bada, orduan ez dela $\geq \kappa^{+4}$ tamainako $\kappa$-PCF espaziorik (ikus [2] eta [14]). Beraz, bereziki ez dago $\geq \omega_{4}$ tamainako $\omega$-PCF espaziorik. Azken emaitza hori erabili zuen gero Shelahk $2^{\varkappa \omega}$-ko $x_{\omega 4}$ bornea lortzeko, baldin eta $x_{\omega}$ muga gogorreko kardinala bada. Halaber, Ruylek frogatu zuen [12]n ZFCrekin konsistentea dela $\omega_{2}$ altuerako $\omega$-PCF espazio bat izatea, eta, hala, ezin dugula espero Shelahren $2^{\varkappa \omega}$-ko bornea $\varkappa_{\omega 2}$-ra hobetzerik, Shelahk erabilitako metodoak erabilita behintzat. Ohartu $\omega_{2}$ altuerako $\omega$-PCF espazio bat altuera bereko $\omega_{1}$-PCF espazio bat ere badela, eta, hala, ezin dugu, ezta ere, espero Shelahren $2^{\varkappa \omega 1}$-eko bornea $x_{\omega 2}$-ra hobetzerik. Gainera, [12]n zehaztu zen kardinalen zein segida izan daitekeen $\omega_{2}$ altuerako $\omega$-PCF espazio baten segida kardinala. Horrez gain, honako emaitza hau lortu zen [3]n: $\eta<\omega_{3}$ ordinal ororentzat ZFCrekin konsistentea da $\eta$ altuerako $\omega$-PCF espazio bat izatea non espazioaren Cantor-Bendixson maila guztiak zenbakarriak diren. Hala ere, honako arazo hau argitzeke dago:

3. galdera. ZFCrekin konsistentea al da $\omega_{3}$ altuerako $\omega$-PCF espazio bat izatea?

3. galderari dagokionez, jakin ere ez dakigu konsistentea ote den $\omega_{3}$ altuerako LCS espazio bat egotea, non espazioaren Cantor-Bendixson maila guztiak zenbakarriak diren. Froga bageneza ZFCn ez dela $\omega_{3}$ altuerako $\omega$-PCF espaziorik, hobetu egingo genuke Sheilahren $2^{\varkappa \omega}$-ko bornea $\varkappa_{\omega 3}$-ra.

Halaber, badirudi honako arazo hau ere argitzeke dagoela:

4. galdera. ZFCrekin konsistentea al da $\omega_{3}$ altuerako $\omega_{2}$-PCF espazio bat izatea? 
4. galderari baietz erantzutea bageneuka, ezingo genuke espero 1. teoremako $2^{\varkappa \omega 2}$-ko bornea $x_{\omega 3}$-ra hobetzerik, PCF teoria baliatuta behintzat. Bestetik, [7]ko emaitza nagusiaren ondorioz, daukagu ZFCrekin koherentea dela $\eta<\omega_{4}$ ordinal ororentzat $\eta$ altuerako LCS espazio bat izatea, non espazioaren Cantor-Bendixson maila guztiek $\omega_{2}$ tamaina duten.

Argitzeke dagoen hurrengo arazoak PCF Aieruarekin du zerikusia. Lehenbizi, nozio topologiko batzuk aurkeztu beharra daukagu. X espazio bat bada eta Y X-ren azpimultzo bat bada, Y-k X-n duen itxitura sekuentziala honela definitzen da: $\lim (\mathrm{Y})=\left\{\mathrm{x} \in \mathrm{X}\right.$ : bada $\left(\mathrm{y}_{\mathrm{n}}\right)_{\mathrm{n}}$ segida bat $\left.\mathrm{Y}-\mathrm{n} \operatorname{non} \lim _{\mathrm{n}} \mathrm{y}_{\mathrm{n}}=\mathrm{x}\right\}$. $\mathrm{X}$ segida sekuentzial bat dela esaten dugu, baldin eta, $\lim (\mathrm{Y})=\mathrm{Y}$-dun $\mathrm{X}$-ren Y azpimultzo ororentzat, Y itxia bada. Todorcevicek erakutsi zuen $\omega$-PCF espazio oro sekuentziala dela, eta teorema horren orokortzea Pereirak lortu zuen [10]en (2. atalean). Bereziki, baldin eta $A=\left\{x_{n+1}: n \in \omega\right\}, X(P C F(A))$ espazioa sekuentziala da. Orobat, X espazioa Fréchet-Urysohn dela diogu, baldin eta X-ren Y azpimultzo ororentzat $\lim (\mathrm{Y})=\overline{\mathrm{Y}}$. Argi dago Fréchet-Urysohn espazio oro sekuentziala dela. Eta [10]en (3. atalean) frogatu zen Continuumaren Hipotesiaren baitan Fréchet-Urysohn ez den $\omega$-PCF espazio bat dagoela. Azken emaitza hori [9]n orokortu zen, non erakutsi zen Continuumaren Hipotesiaren azpian badaudela Fréchet-Urysohn ez diren $\omega_{2}$ binakako $\omega$-PCF espazio ez-homeomorfikoak. Nolanahi ere, badirudi argitzeke dirauela Todorcevici zor zaion honako galdera honek.

5. galdera. Izan bedi $A=\left\{x_{n+1}: n \in \omega\right\}$. X(PCF(A)) espazioa FréchetUrysohn al da?

Erraza da ikustea A kardinal erregularren tarte progresibo zenbakarri bat bada, non PCF(A) ere zenbakarria den, orduan X(PCF(A)) espazioak oinarri zenbakarri bat duela, eta, beraz, Fréchet-Urysohn dela. Hortaz, baldin eta $\operatorname{PCF}\left(\left\{x_{n+1}: n \in \omega\right\}\right)$-ri lotutako espazioa ez bada Fréchet-Urysohn, Shelahren PCF Aierua faltsua litzateke.

\section{Erreferentzia bibliografikoak}

[1] Abraham, Uri \& Magidor, Menachem (2010), «Cardinal arithmetic». In Matthew Foreman \& Akihiro Kanamori (arg.), Handbook of Set Theory, 2. alea. New York: Springer, 1149-1227.

[2] Burke, Maxim R. \& MAgidor, Menachem (1990), «Shelah's pcf theory and its applications». Annals of Pure and Applied Logic 50 (3): 207-254.

[3] Er-Rhaimini, Karim \& VeličKović, Boban (2010), «PCF structures of height less than $\omega_{3} »$. The Journal of Symbolic Logic 75 (4): 1231-1248.

[4] Foreman, Matthew (2005), «Some problems in singular cardinals combinatorics». Notre Dame Journal of Formal Logic 46 (3): 309-322.

[5] GiTiK, Moti \& Magidor, Menachem (1992), «The singular cardinal hypothesis revisited». In Haim Judah, Winfried Just \& Hugh Woodin (arg.), Set Theory of the 
Continuum. Mathematical Sciences Research Institute Publications, 26. liburukia. New York: Springer, 243-279.

[6] GitiK, Moti \& SHelah, Saharon (2013), «Applications of pcf for mild large cardinals to elementary embeddings». Annals of Pure and Applied Logic 164 (9): 855865.

[7] MarTínEZ, Juan C. (1999), «A forcing construction of thin-tall Boolean algebras». Fundamenta Mathematicae 159 (2): 99-113.

[8] Martínez, Juan C. (2014), «Cardinal sequences for superatomic Boolean algebras». In Stefan Geschke, Benedikt Löwe \& Philipp Schlicht (arg.), Infinity, Computability and Metamathematics. Tributes Series, 23. liburukia. Milton Keynes: College Publications, 273-284.

[9] MARTínez, Juan C. (argitaratzeko), «On PCF spaces which are not Fréchet-Urysohn». Reports on Mathematical Logic.

[10] Pereira, Luís (2008), «Applications of the topological representation of the pcfstructure». Archive for Mathematical Logic 47 (5): 517-527.

[11] Pereira, Luís (2008), «The PCF Conjecture and large cardinals». The Journal of Symbolic Logic 73 (2): 674-688.

[12] RuYLe, Jonathan C. (1999), Cardinal sequences of PCF structures. Doktoretza-tesia, University of California, Riverside.

[13] Shelah, Saharon (1992), «Cardinal arithmetic for skeptics». Bulletin of the American Mathematical Society (New Series) 26 (2): 197-210.

[14] SHeLAH, Saharon (1994), Cardinal arithmetic. Oxford Logic Guides, 29. liburukia. Oxford University Press. 